\title{
Radical Uncertainty: Beyond Probabilistic Models of Belief
}

\author{
Jan-Willem Romeijn • Olivier Roy
}

Received: 30 September 2014/ Accepted: 30 September 2014/Published online: 16 October 2014

(C) Springer Science+Business Media Dordrecht 2014

Over the past decades or so the probabilistic model of rational belief has enjoyed increasing interest from researchers in epistemology and the philosophy of science. Of course, such probabilistic models were used for much longer in economics, in game theory, and in other disciplines concerned with decision making. Moreover, Carnap and co-workers used probability theory to explicate philosophical notions of confirmation and induction, thereby targeting epistemic rather than decisiontheoretic aspects of rationality. However, following Carnap's early applications, philosophy has more recently seen an increased popularity of probabilistic models in other areas concerned with the philosophical analysis of belief: there are models targeting coherence, informativeness, simplicity, and so on.

In brief, the probabilistic model of belief comprises of a language, detailing the propositions about which an agent is supposed to have beliefs, and a function over the language that expresses beliefs: the strength of a belief in a proposition is expressed by means of a probability, i.e., a real number between 0 and 1, assigned to that probability. The model offers several rules on how to revise beliefs, of which Bayes' rule is the most prominent. It will not come as a surprise that philosophers have enquired into the adequacy of this model, as a means of representing the various concerns and positions in their debate. The philosophical analysis of belief

\footnotetext{
J.-W. Romeijn ( $\bowtie)$

University of Groningen, Groningen, The Netherlands

e-mail: j.w.romeijn@rug.nl

J.-W. Romeijn

University of Johannesburg, Johannesburg, South Africa

O. Roy

University of Bayreuth, Bayreuth, Germany
} 
offers subtle distinctions and categories, and it is by no means clear that the probabilistic model is versatile enough to accommodate all of these.

Indeed, the model has a number of fixities. One very important limitation is that the language in which beliefs are expressed, is not amenable to revisions. This would involve setting up a new probabilistic model. Another important limitation is that the model automatically constrains probability assignments to comply to logical relations between propositions. But that is at odds with how rational agents deal with logical relations: being rational does not entail that one is logically omniscient.

For present purposes, however, the central shortcoming of the probabilistic model is that, at first sight at least, it cannot express the epistemic state of radical uncertainty. With the notion of radical uncertainty we might mean a number of things. For one, we could be referring to a state of utter cluelessness, in which we have no language to express what we are uncertain about. We can also mean a state of, what may be called, model uncertainty, in which we doubt our modelling assumptions but have insufficient means in the model to express alternative assumptions. And radical uncertainty may refer to an epistemic state in which we have insufficient grasp of our uncertainty regarding a distinct set of propositions.

All these notions of radical uncertainty are in some sense under scrutiny in the current special issue. There are papers by Richard Bradley and Mareile Drechsler, by Jon Williamson, by Jake Chandler, by Seamus Bradley and Katie Steele, and by Arthur Paul Pedersen and Gregory Wheeler, in that order. The first paper, by Bradley and Drechsler, tackles the issue of radical uncertainty directly. They consider a variety of types of uncertainty that go beyond "empirical uncertainty", i.e., the one usually expressed in terms of probability. They show how these other types of uncertainty cannot, without further costs in severity, be captured by the usual probabilistic model. This paper offers an attractive overview of issues brought on by radical uncertainty.

To express radical uncertainty of a particular kind, we might employ so-called imprecise probability assignments: rather than a single, sharp probability function over the propositions at hand, our uncertainty can be expressed by a set of such probability functions. The papers by Williamson and Chandler concern, roughly speaking, the question of how we might motivate sharp or imprecise probability functions as expressions of belief. Williamson considers how we can motivate sharp values for our probability assignments in order to put a stop to a regress into ever higher orders of uncertainty. He finds-perhaps unsurprisingly-that objective Bayesianism holds the best promise for a proper motivation of that. Chandler's paper, by contrast, criticizes an argument in favour of representing uncertainty by means of sharp probability values, showing that it is based on an unnecessarily strict principle concerning rational choice.

The last two papers of this issue are devoted to an analysis of a phenomenon that troubles the use of imprecise probability assignments as representations of belief: dilation. The challenging version of this phenomenon is that a sharp probability assignment to a proposition might be updated to an imprecise one, possibly spanning the full range from 0 to 1 , upon learning any one of the elements from an information partition that seemingly bears no relevance to the proposition in question. Bradley and Steele investigate several resolutions to this problem, 
exploring variations on how probability assignments are updated upon learning new information, and considering restrictions on prior probability assignments that help to avoid dilation altogether. Pedersen and Wheeler, finally, approach the phenomenon of dilation from a more mathematical angle. They distinguish between proper and improper dilation, and trace the phenomenon back to particular failures of stochastic independence among propositions. They thereby defuse the more spectacular instances of dilation, and make a case for accepting the less spectacular versions as a fact of epistemic life.

Taken together, the five papers in this special issue offer a good view of current philosophical work on how to represent uncertainty. One view that all the authors seem to share is that imprecise probability assignments present a serious alternative to the standard probabilistic model of belief. We believe that formal approaches to epistemology and philosophy of science will, in the next few decades, benefit greatly from imprecise probabilism. The cult following that imprecise probabilists have had in the past might well develop into popular philosophical culture.

Acknowledgments The authors wish to thank the Netherlands Organization of Scientific Research (NWO) and the fellowship programme of the University of Johannesburg for financial support. The idea of making a special issue on radical uncertainty was conceived in the context of a European research network on Rationality and Decision funded by the Netherlands organisation for scientific research (NWO), and supported by grant programmes running at several other institutions (London School of Economics, Ludwig Maximilians University Munich, University of Leuven). The papers in this special issue were all presented at a workshop in Munich in 2012. More on the activities of our research network can be found online at http://www.philos.rug.nl/R\&Dnetwork/. 